\title{
Denbinobin, a phenanthrene from Dendrobium nobile, inhibits invasion and induces apoptosis in SNU-484 human gastric cancer cells
}

\author{
JAE IN SONG $^{1 *}$, YU JIN KANG ${ }^{1 *}$, HAE-YOUNG YONG ${ }^{1}$, YOUNG CHOONG KIM $^{2}$ and AREE MOON ${ }^{1}$ \\ ${ }^{1}$ College of Pharmacy, Duksung Women's University, 419 Ssangmun-Dong, Seoul 132-714; ${ }^{2}$ College of Pharmacy \\ and Research Institute of Pharmaceutical Science, Seoul National University, Seoul 151-742, Republic of Korea
}

Received August 31, 2011; Accepted October 17, 2011

DOI: $10.3892 /$ or.2011.1551

\begin{abstract}
Dendrobium nobile is widely used as an analgesic, an antipyretic, and a tonic to nourish the stomach in traditional medicine. Mounting evidence suggests an antitumor activity of denbinobin, a major phenanthrene isolated from stems of Dendrobium nobile. The present study aimed to investigate the inhibitory effect of denbinobin on the invasive ability of human cancer cells. The cytotoxicity of denbonobin was examined in several human cancer cell lines including SK-Hep-1 hepatocarcinoma cells, SNU-484 gastric cancer cells, and HeLa cervix cancer cells. Because SNU-484 cells showed the lowest $\mathrm{IC}_{50}$ value, we examined the effect of denbinobin on the invasive ability of SNU-484 cells. The present study revealed, for the first time, that denbinobin inhibits the invasive phenotype of SNU-484 human gastric cancer cells in a dose-dependent manner. Expressions of matrix metalloproteinase (MMP)-2 and MMP-9 were significantly decreased by denbinobin, suggesting that MMP-2/-9 may be responsible for the antiinvasive activity of denbinobin. We also provide evidence that denbinobin induces apoptosis through down-regulation of Bcl-2 and an up-regulation of Bax. Taken together, this study demonstrates that denbinobin inhibits invasion and induces apoptosis in highly invasive SNU-484 human gastric cancer cells. Given that gastric cancer has been estimated to be one of the most common causes of cancer-related death among Asians and the major cause of death from gastric cancer is the metastatic spread of the disease, our findings may provide useful information regarding the application of denbinobin as a chemopreventive agent that could prevent or alleviate metastatic gastric cancer.
\end{abstract}

Correspondence to: Dr Aree Moon, College of Pharmacy, Duksung Women's University, 419 Ssangmun-Dong, Tobong-Ku, Seoul 132-714, Republic of Korea

E-mail: armoon@duksung.ac.kr

${ }^{*}$ Contributed equally

Key words: denbinobin, gastric cancer, invasion, apoptosis, S100A8

\section{Introduction}

Dendrobium nobile Lindl. (Orchidaceae, also called 'Seokgok' in Korea; 'Shi Hum' in mainland China) has been used as an analgesic, an antipyretic, and a tonic to nourish the stomach in traditional medicine (1). We have previously isolated denbinobin (5-hydroxy-3,7-dimethoxy-1,4-phenanthraquinone) as one of the antifibrotic phenanthrenes from the stems of $D$. nobile (2). Denbinobin has been demonstrated to induce cell death in various human cancer cells including leukemic cells (3-5), lung adenocarcinoma cells (6), and human colorectal cancer cells (7).

Metastasis is a characteristic of highly malignant cancers with poor clinical outcome. Metastasis is a multi-step process involving detachment of malignant transformed cells from the primary tumor site, attachment to the extracellular matrix (ECM), degradation of the ECM and basement membrane (invasion), and cell migration through the degraded matrix (8). A recent study showed that denbinobin suppresses invasion in mouse breast tumor cells in vitro and metastasis of breast cancer in a mouse metastasis model in vivo (9). Tumor invasion is often associated with the enhanced synthesis and/or activation of matrix-degrading enzymes matrix metalloproteinases (MMPs), among which MMP-2 and MMP-9 are of central importance (10-12).

The differentiated cells of multicellular organisms share the ability to carry out their own death through activation of a programmed cell suicide process known as apoptosis (13). Apoptotic cell death is regulated by members of the caspase family and Bcl-2 family (14,15). Caspase family members consist of cysteine proteases which play a critical role in apoptosis. Especially, caspase-3 is responsible for the proteolytic cleavage of many proteins related to apoptosis (16). Caspase- 3 is initially produced as a pro-caspase- $3(32 \mathrm{kDa})$ and then activated by cleavage events to generate a large subunit of $17 \mathrm{kDa}$ and a small subunit of $12 \mathrm{kDa}(17)$. Bcl-2 family proteins modulate mitochondria-dependent apoptosis through the balance of antiand pro-apoptotic members and regulate processes involved in the life and death of cells $(14,18)$. The anti-apoptotic protein Bcl-2 prevents apoptotic death, while the death promoting proapoptotic protein Bax counteracts the anti-apoptotic effects of $\mathrm{Bcl}-2$ by forming a heterodimer with Bcl-2. 


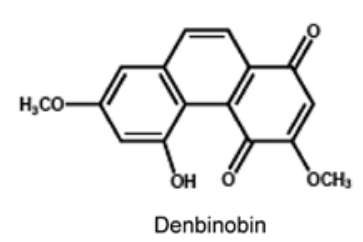

Figure 1. Chemical structure of denbinobin $(2,36)$.

Although mounting evidence suggests an antitumorigenic effect of denbinobin (1), the role of denbinobin in the metastatic potential of cancer cells has not been extensively studied. In the present study, we investigated the effect of denbinobin on the invasive phenotype of the SNU-484 human gastric cancer cell line. Here, we show that denbinobin efficiently inhibits the invasive ability of SNU-484 cells in a dose-dependent manner, possibly via down-regulation of MMP-2 and MMP-9. We further show that denbinobin induces apoptosis through a down-regulation of Bcl-2 and an up-regulation of Bax.

\section{Materials and methods}

Materials. Denbinobin was isolated from a methanolic extract of Dendrobium nobile stems as previously described (2). The chemical structure of denbinobin is depicted in Fig. 1.

Cell lines. The SK-Hep-1 hepatocarcinoma cell line (KCLB no. 30052), and the HeLa cervical adenocarcinoma cell line (KCLB no. 10002) were purchased from the Korean Cell Line Bank (KCLB, Seoul, Korea). The SNU-484 gastric cancer cell line was kindly provided by Dr H.D. Um (Korea Institute of Radiological and Medical Science, Seoul, Korea). SK-Hep-1 and HeLa cells were cultured in DMEM medium supplemented with $10 \%$ heat-inactivated fetal bovine serum and $100 \mathrm{U} / \mathrm{ml}$ penicillin-streptomycin. SNU-484 cells were cultured in RPMI-1640 supplemented with $10 \%$ heat-inactivated fetal bovine serum and $50 \mathrm{U} / \mathrm{ml}$ gentamicin. Cells were maintained in a humidified atmosphere with $95 \%$ air and $5 \%$ $\mathrm{CO}_{2}$ at $37^{\circ} \mathrm{C}$.

3-(4,5-Dimethylthiazol-2-yl)-2,5-diphenyltetrazolium bromide $(M T T)$ assay. Cells $\left(5 \times 10^{3}\right)$ cultured in a 96-well plate were treated with denbinobin for $24 \mathrm{~h}$. After $24 \mathrm{~h}$ of incubation, $25 \mu \mathrm{l}$ of $0.5 \mathrm{mg} / \mathrm{ml}$ of MTT was added and incubation was continued for another $4 \mathrm{~h}$. Conversion of MTT to purple formazan by metabolically active cells indicated the extent of cell viability. The crystals of produced formazan were dissolved with $100 \mu \mathrm{l}$ of DMSO and the optical density was measured at $540 \mathrm{~nm}$ using a micro-ELISA reader (Molecular Devices, Sunnyvale, CA, USA) for quantification of cell viability. Assays were performed in triplicate.

Flow cytometry (fluorescence-activated cell sorting, FACS). SNU-484 cells were incubated with $10 \mu \mathrm{M}$ denbinobin for $4 \mathrm{~h}$, and then harvested and washed with cold PBS. Apoptotic cells were quantitatively identified with the Annexin V-FITC Apoptosis Detection kit I (BD Biosciences Pharmingen, San Diego, CA, USA). The cells were then resuspended in binding buffer at a concentration of $1 \times 10^{6}$ cells $/ \mathrm{ml}$. Resuspended cells $\left(8 \times 10^{4}\right.$ cells $\left./ 100 \mu \mathrm{l}\right)$ were stained with $4 \mu \mathrm{l}$ of FITC-conjugated
Annexin-V and $4 \mu \mathrm{l}$ of propidium iodide reagent. After incubation in the dark for $15 \mathrm{~min}$ at room temperature, $0.4 \mu \mathrm{l}$ of binding buffer was added to each tube. The cells were sorted by flow cytometry (Beckman-Coulter, Miami, FL, USA).

In vitro invasion assay. An in vitro invasion assay was performed using a 24-well transwell unit with polycarbonate filters (Corning Costar, Cambridge, MA, USA) as previously described (19). The lower side of the filter was coated with type I collagen, and the upper side was coated with Matrigel (Collaborative Research, Lexington, KY, USA). The lower compartment was filled with serum-free media containing $0.1 \%$ BSA. Cells were placed in the upper part of the Transwell plate, incubated for $17 \mathrm{~h}$, fixed with methanol, and stained with hematoxylin for $10 \mathrm{~min}$, followed by a brief staining with eosin. The cells on the upper surface of the filter were removed using a cotton swab. The invasive phenotypes were determined with microscopy at $x 400$ by counting the cells that invaded the lower side of the filter. Thirteen fields were counted for each filter and each sample was assayed in triplicate.

Gelatin zymography assay. Cells were cultured in serum-free medium for $48 \mathrm{~h}$. Gelatinolytic activity of the conditioned media was determined by the gelatin zymography assay as previously described (20). Areas of gelatinase activity were detected as clear bands against the blue-stained gelatin background. Relative band intensities were determined by quantitation of each band with an image analyzer Gel Logic 200 imaging system (Kodak, Rochester, NY, USA).

Immunoblot analysis. Immunoblot analysis was performed as previously described (21) using anti-S100A8, anti-S100A9, anti-Bax, anti-Bcl-2, anti-caspase-3, anti-JNK, and anti-phosphorylated JNK antibodies from Santa Cruz Biotechnology Inc. (Santa Cruz, CA, USA); anti-p38, anti-phosphorylated p38, anti-ERK and anti-phosphorylated ERK antibodies from Cell Signaling Technology (Beverly, MA, USA). The enhanced chemiluminescence (ECL, Amersham-Pharmacia, USA) system was used for detection. Relative band intensities were determined with an image analyzer (Gel Logic 200 imaging system).

\section{Results}

Denbinobin inhibits the proliferation of gastric cancer cells. The cytotoxic effect of denbonobin was examined in several human cancer cell lines by the MTT assay. Treatment with denbinobin for $24 \mathrm{~h}$ inhibited the growth of SK-Hep-1 hepatocarcinoma cells, SNU-484 gastric cancer cells, and HeLa cervix cancer cells in a dose-dependent manner with the $\mathrm{IC}_{50}$ values of 16.4, 7.9 and $22.3 \mu \mathrm{M}$, respectively (Fig. 2). Because SNU-484 gastric cancer cells showed the lowest $\mathrm{IC}_{50}$ value among the cancer cell lines tested, we further examined the effect of denbinobin on the invasive ability of SNU-484 cells.

Denbinobin inhibits the invasive phenotype of SNU-484 gastric cancer cells. An in vitro invasion assay was conducted to investigate the effect of denbinobin on the invasive phenotype of SNU-484 cells. As shown in Fig. 3A, invasiveness of SNU-484 cells was significantly inhibited by denbinobin 
A

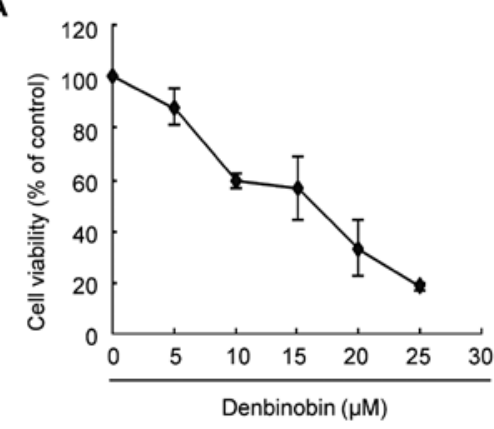

B

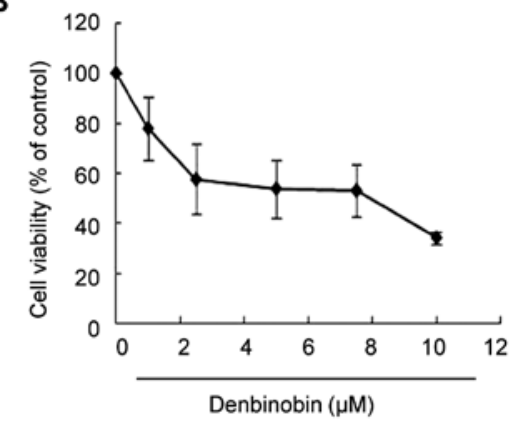

C

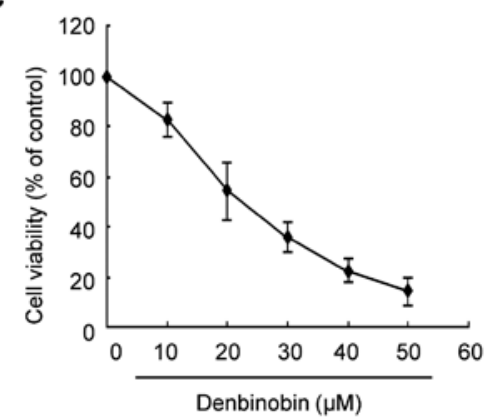

Figure 2. Denbinobin inhibits proliferation of cancer cells. Cells were treated with various concentrations of denbinobin for $24 \mathrm{~h}$ and subjected to MTT assay. (A) SK-Hep-1 hepatocarcinoma cells (B) SNU-484 gastric cancer cells (C) HeLa cervical adenocarcinoma cells. The results represent the mean \pm SE of triplicates.

in a dose-dependent manner. Treatment of SNU-484 cells with $2.5 \mu \mathrm{M}$ denbinobin for $17 \mathrm{~h}$ inhibited the invasiveness of SNU-484 cells by $50 \%$. These results demonstrate that denbinobin efficiently inhibits the invasive ability of SNU-484 gastric cancer cells.

Denbinobin inhibits the expression of MMP-2 and MMP-9. Tumor invasion often involves enhanced synthesis and secretion of MMP-2 and MMP-9. We next examined the effect of denbinobin on the expression of MMP-2 and/or MMP-9 in SNU-484 cells. As shown in Fig. 3B, the levels of expression of both MMP-2 and MMP-9 were dose-dependently decreased by denbinobin. These data imply that the inhibitory effect of denbinobin on the invasiveness of SNU-484 cells may be due to the down-regulation of MMP-2 and MMP-9.

Denbinobin inhibits the expression of S100A8. The S100 proteins are calcium-binding proteins involved in cell growth and migration, among which, S100A8 and S100A9 are implicated in tumor development and/or progression (22-24). Our previous study indicated that S100A8 and S100A9 are critical
A

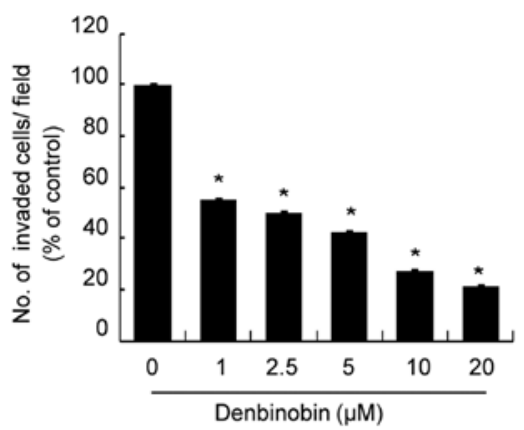

B
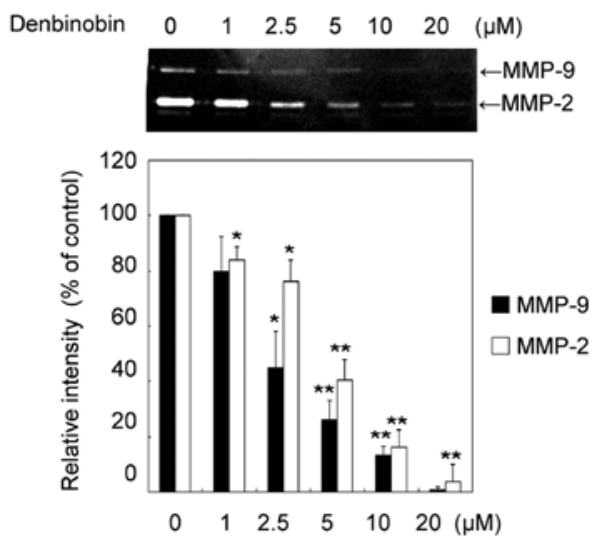

C
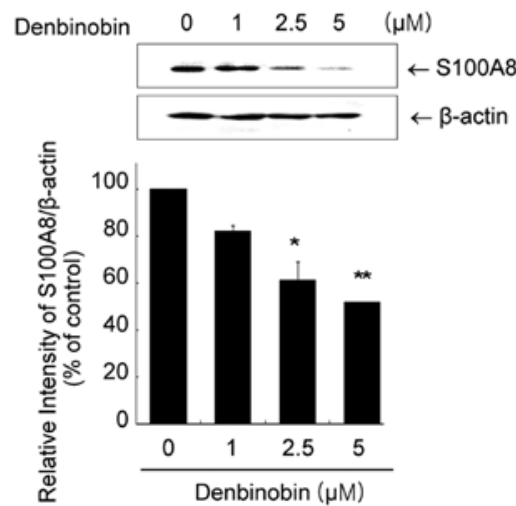

Figure 3. Denbinobin inhibits the invasive phenotype of SNU-484 gastric cancer cells. (A) SNU-484 cells were treated with various concentrations of denbinobin and subjected to the in vitro invasion assay. The number of invaded or migrated cells per field was counted (x400) in 13 arbitrary visual fields. "Statistically different from control at $\mathrm{p}<0.01$. (B) The SNU-484 cells were treated with various concentrations of denbinobin for $48 \mathrm{~h}$. The conditioned media were collected $(50 \mu \mathrm{g})$ and the gelatinolytic activities of secreted MMP-2 and MMP-9 were determined by gelatin zymogram assay. Relative band intensities were determined by an image analyzer. The black bar represents MMP-9, and the white represents MMP-2.,"** Statistically different from control at $\mathrm{p}<0.05$ and $\mathrm{p}<0.01$, respectively. (C) SNU-484 cells were treated with various concentrations of denbinobin for $24 \mathrm{~h}$. Cell lysates were prepared and immunoblot analysis was performed to detect S100A8. Relative band intensities were determined by quantitation of each band by an image analyzer. The results presented are the mean \pm SE of triplicates. ${ }^{* * *}$ Statistically different from control at $\mathrm{p}<0.05$ and $\mathrm{p}<0.01$, respectively.

for the invasive and migratory phenotypes of SNU-484 cells in which MMP-2 is involved (25). We next examined the effect of denbinobin on the expressions of S100A8/A9 in SNU-484 cells. Treatment with denbinobin inhibited the expression of 
A

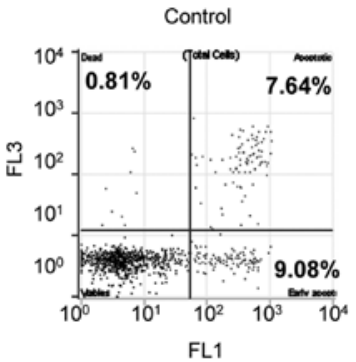

B
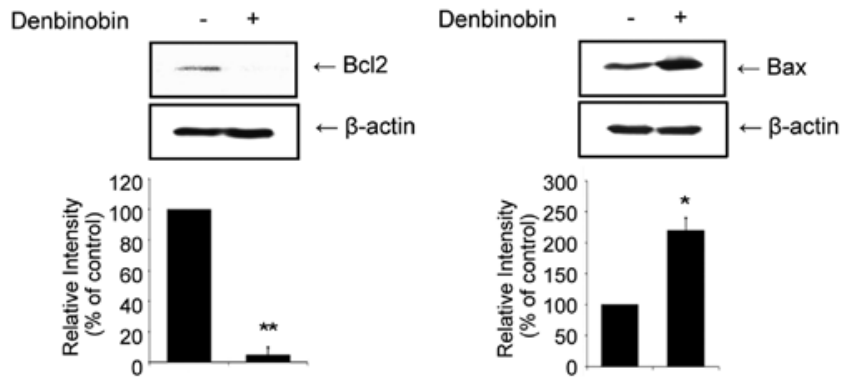

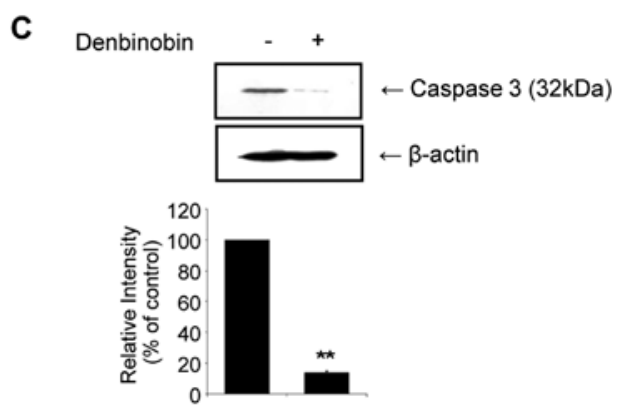

Figure 4. Denbinobin induces apoptosis through a MAPK-dependent pathway. (A) SNU-484 cells were incubated with either DMSO or with $10 \mu \mathrm{M}$ denbinobin for $4 \mathrm{~h}$. FACS analysis determined the incidence of apoptosis in SNU-484 cells treated with DMSO or with $10 \mu \mathrm{M}$ denbinobin and stained with propidium iodide and Annexin-V. (B and C) Cells were incubated with denbinobin $(10 \mu \mathrm{M})$ for $24 \mathrm{~h}$. Total cell extracts were analyzed by immunoblot analysis with specific antibodies. Relative band intensities were determined by quantitation of each band by an Image analyzer. The results presented are the mean \pm SE of triplicates.

${ }^{*, * *}$ Statistically different from control at $\mathrm{p}<0.05$ and $\mathrm{p}<0.01$, respectively.

S100A8 in a dose-dependent manner (Fig. 3C), while expression of S100A9 was not affected (data not shown).

Denbinobin induces apoptosis in gastric cancer cells. FACS analysis was conducted to investigate whether the denbinobininduced growth inhibition involves apoptosis. As shown in Fig. 4A, treatment of SNU-484 cells with $1 \mu \mathrm{M}$ denbinobin for $4 \mathrm{~h}$ resulted in a marked increase in Annexin-positive apoptotic cells (17.98\%) compared with the control cells (7.64\%). These results imply that the observed growth inhibitory effect of denbinobin may be due to the induction of apoptotic cell death.

Because proteins of the Bcl-2 family are suggested to be involved in the control of apoptosis (26), we further investigated the effect of denbinobin on the expressions of Bcl-2 and Bax. A prominent decrease of $\mathrm{Bcl}-2$ was observed in the SNU-484 cells treated with $10 \mu \mathrm{M}$ denbinobin for $24 \mathrm{~h}$, while Bax was markedly increased by the same treatment (Fig. 4B). The data demonstrate that denbinobin induces apoptosis by down-regulation of the anti-apoptotic protein Bcl-2 and up-regulation of the pro-apoptotic protein Bax in SNU-484 cells. We also examined the effect of denbinobin on the degradation of pro-caspase-3. As shown in Fig. 4C, the level of pro-caspase-3 was markedly decreased by denbinobin treatment, suggesting that denbinobin induces activation of caspase-3 in SNU-484 cells.

Denbinobin activates ERKs and p38 MAPK. Denbinobin was shown to induce sustained activation of the ERKs, p38 MAPK, and JNK in human leukemic cells (5). Kinetic studies were conducted to investigate the involvement of these signaling molecules in denbinobin-induced growth inhibition of SNU-484 cells. As shown in Fig. 5A, a drastic increase in the level of phosphorylated p38 MAPK was observed with denbinobin treatment in a time-dependent manner. Denbinobin also activated ERKs, but to a lesser degree than p38 MAPK. Activation of JNK was not affected by denbinobin (Fig. 5A).

We then examined the role of p38 MAPK and ERKs on denbinobin-induced pro-caspase-3 degradation using pharmacological inhibitors of these signaling molecules. As shown in Fig. 5B, pretreatment of SNU-484 cells with SB203085, a p38 MAPK inhibitor, and PD98059, an inhibitor of MEK which is an ERK activator, significantly reversed the denbinobininduced pro-caspase- 3 degradation. These results indicate the possible involvement of $\mathrm{p} 38$ MAPK and ERKs pathways in denbinobin-induced apoptosis in SNU-484 gastric cancer cells. 
A

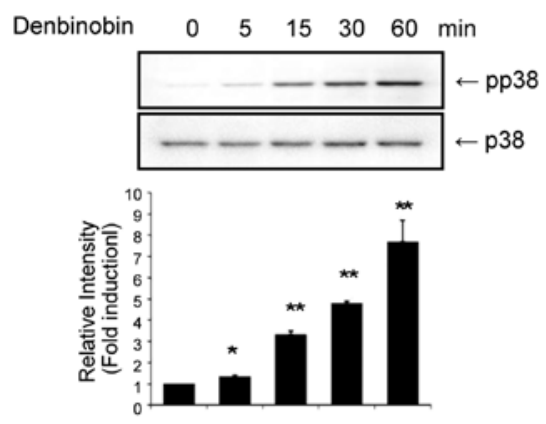

$\begin{array}{lllllll}\text { Denbinobin } & 0 & 5 & 15 & 30 & 60 & \mathrm{~min}\end{array}$

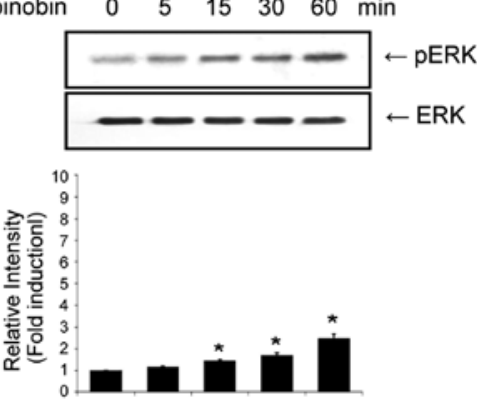

Denbinobin $\quad 0 \quad 5 \quad 15 \quad 30 \quad 60 \quad \mathrm{~min}$

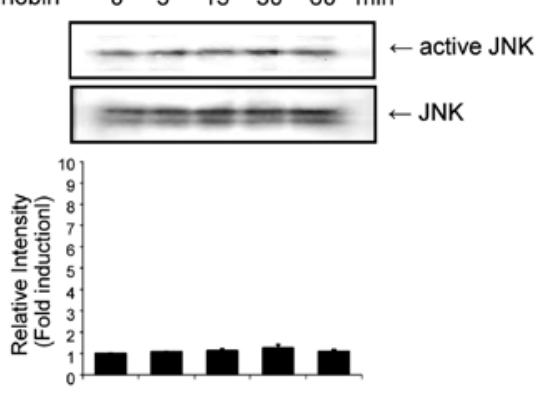

B

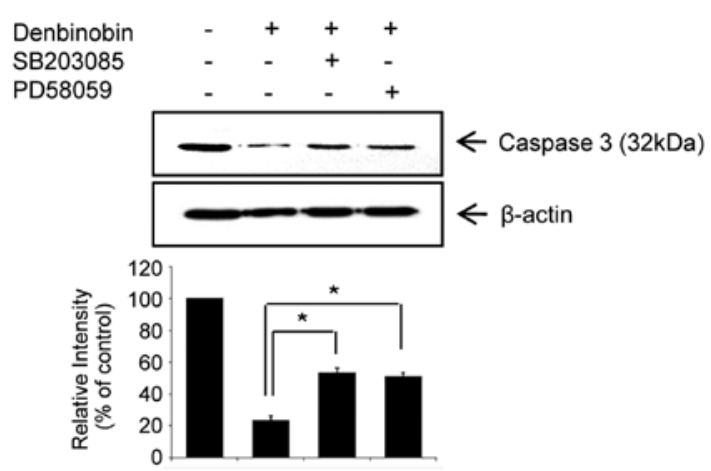

Figure 5. Denbinobin induces apoptosis through a MAPK-dependent pathway. (A) SNU-484 cells were incubated with denbinobin $(10 \mu \mathrm{M})$ during the indicated times and phosphorylated p38, ERK and phosphorylated JNK were analyzed using specific antibodies by immunoblot analysis. (B) Cells were pretreated with SB203580 $(10 \mu \mathrm{M})$ and PD98059 $(10 \mu \mathrm{M})$ for $30 \mathrm{~min}$ and then treated with denbinobin $(10 \mu \mathrm{M})$ for $24 \mathrm{~h}$. Caspase- 3 was detected by immunoblot analysis. Relative band intensities were detected by quantitation of each band by an Image analyzer. The results presented are the mean $\pm \mathrm{SE}$ of triplicates. ${ }^{*}{ }^{* * *}$ Statistically different from control at $\mathrm{p}<0.05$ and $\mathrm{p}<0.01$, respectively.

\section{Discussion}

Many studies have focused on the anti-carcinogenic and/or chemopreventive activities of phytochemicals. While many studies have reported the anti-proliferative effect of denbinobin in a variety of cancer cells, there has been a limited amount of information on the anti-invasive activity of denbinobin. In the present study, we provide evidence, for the first time, that denbinobin inhibits the invasive phenotype of a highly invasive cell line; the SNU-484 human gastric carcinoma cell line. Because metastasis is one of the major causes of mortality in gastric cancer patients, inhibition of invasion and metastasis of cancer cells is of great significance for the treatment of gastric cancer (27). One of the major drawbacks for anti-cancer agents are the unwanted side effects. Taken in conjunction with the fact that $D$. nobile has been widely used as an oriental medicine for many years (1), the adverse effects of denbinobin should not be significant.

Numerous studies have shown a correlation between the levels of MMP-2 and/or MMP-9 and the invasive phenotype of cancer cells. An imbalance between proteinases and their inhibitors has been demonstrated to be a key factor in many human diseases, especially tumorigenesis (28). Several laboratories, including ours, have shown that among MMPs, MMP-2 expression is related to gastric cancer invasion and metastasis $(25,29,30)$. The present study demonstrates that denbinobin exerts an inhibitory effect on the invasiveness of SNU-484 cells, which may be associated with down-regulation of both MMP-2 and MMP-9. It is worthwhile to further investigate the molecular mechanisms underlying the possible contribution of MMP-2/-9 to denbinobin-induced inhibition of gastric cancer cell invasion.

Recent reports have suggested the association of S100A8 and S100A9 with cell migration in several cancer cell lines $(31,32)$. Our previous studies identified S100A8 and S100A9 as candidate markers for cell invasion in breast epithelial cells (33) and showed that these proteins up-regulated MMP-2, causing invasive phenotype in SNU-484 cells (25). The results obtained from this study demonstrated a dose-dependent down-regulation of S100A8 by denbinobin in SNU-484 cells, suggesting the involvement of S100A8 in the denbinobininduced invasive phenotype. Further studies would be required to elucidate the role of S100A8 in the inhibitory effect of denbinobin on the invasive phenotype of gastric cancer cells.

Apoptosis is arguably the most potent natural defense against cancer (13). Thus, efforts have been made to develop strategies that trigger apoptosis in malignant cancer cells. In this study, we showed that denbinobin induces apoptosis in SNU-484 cells with a prominent decrease of Bcl-2, which prevents apoptosis by blocking the release of cytochrome $\mathrm{C}$ from mitochondria (34). In contrast, Bax, which directly induces cytochrome $\mathrm{C}$ release from mitochondria and thereby triggers caspase activation (35), was increased by denbinobin. The results suggest that denbinobin-induced apoptosis of gastric cancer cells is mediated by Bcl-2 and Bax.

Activation of mitogen-activated protein kinases (MAPKs) including JNK, ERKs, and p38 MAPK, are important intermediates in the signal-transduction pathway associated with cell proliferation and apoptosis (36-38). A recent study showed that treatment of human leukemic cells with denbinobin induced apoptotic signaling and activation of the ERKs, p38 MAPK, and JNK (5). In the present study, we demonstrated that denbinobin activated ERKs and p38 MAPK, but not JNK in SNU-484 cells, suggesting that these signaling molecules 
may mediate denbinobin-induced apoptosis in human gastric cancer cells.

Taken together, the present study clearly demonstrated that denbinobin effectively inhibits cell growth and the invasive phenotype of SNU-484 human gastric cancer cells. Given that metastatic gastric cancer is one of the most lethal malignancies in Korea (39), our novel observation allows us to suggest a potential application of denbinobin as a chemopreventive agent with an anti-invasion effect on gastric cancer patients.

\section{Acknowledgements}

This study was supported by the National Research Foundation of Korea (NRF) grant funded by the Korean government (no. ROA-2008-000-20070-0 and no. R11-20100001707).

\section{References}

1. Kong JM, Goh NK, Chia LS and Chia TF: Recent advances in traditional plant drugs and orchids. Acta Pharmacol Sin 24: 7-21, 2003.

2. Yang HY, Sung SH and Kim YC: Antifibrotic phenanthrenes of Dendrobium nobile stems. J Nat Prod 70: 1925-1929, 2007.

3. Huang YC, Guh JH and Teng CM: Denbinobin-mediated anticancer effect in human K562 leukemia cells: role in tubulin polymerization and Bcr-Abl activity. Biomed Sci 12: 113-121, 2005.

4. Yang KC, Uen YH, Suk FM, Liang YC, Yang YJ, Ho YS, Li IH and Lin SY: Molecular mechanisms of denbinobin-induced antitumorigenesis effect in colon cancer cells. World J Gastroenterol 11: 3040-3045, 2005.

5. Sánchez-Duffhues G, Calzado MA, de Vinuesa AG, Appendino G, Fiebich BL, Loock U, Lefarth-Risse A, Krohn K and Muñoz E: Denbinobin inhibits nuclear factor-kappaB and induces apoptosis via reactive oxygen species generation in human leukemic cells. Biochem Pharmacol 77: 1401-1409, 2009.

6. Kuo CT, Hsu MJ, Chen BC, Chen CC, Teng CM, Pan SL and Lin $\mathrm{CH}$ : Denbinobin induces apoptosis in human lung adenocarcinoma cells via Akt inactivation, Bad activation, and mitochondrial dysfunction. Toxicol Lett 177: 48-58, 2008.

7. Chen TH, Pan L, Guh JH, Chen CC, Huang YT, Pai HC and Teng CM: Denbinobin induces apoptosis by apoptosis-inducing factor releasing and DNA damage in human colorectal cancer HCT-116 cells. Naunyn Schmiedebergs Arch Pharmacol 378: 447-457, 2008

8. Stetler-Stevenson WG, Liotta LA and Kleiner DE Jr: Extracellular matrix 6: role of matrix metalloproteinases in tumor invasion and metastasis. FASEB J 7: 1434-1441, 1993.

9. Chen PH, Peng CY, Pai HC, Teng CM, Chen CC and Yang CR: Denbinobin suppresses breast cancer metastasis through the inhibition of Src-mediated signaling pathways. J Nutr Biochem 22: 732-740, 2011

10. Liotta LA, Abe S, Robey PG and Martin GR: Preferential digestion of basement membrane collagen by an enzyme derived from a metastatic murine tumor. Proc Natl Acad Sci USA 76: 2268-2272, 1979.

11. Steller-Stevenson WG: Type IV collagenases in tumor invasion and metastasis. Cancer Metastasis Rev 9: 289-303, 1990.

12. Tryggvason K: Type IV collagenases in invasive tumors. Breast Cancer Res Treat 24: 209-218, 1993.

13. Raff MC: Social control on cell survival and cell death. Nature 356: 397-400, 1992

14. Tsujimoto Y and Shimizu S: Bcl-2 family: life-or-death switch. FEBS Lett 466: 6-10, 2000.

15. Tsujimoto Y: Bcl-2 family of proteins: life-or-death switch in mitochondria. Biosci Rep 22: 47-58, 2002.

16. Cohen GM: Caspases: the executioners of apoptosis. Biochem J 1: $1-16,1997$.

17. Tewari M, Tewari M, Quan LT, O'Rourke K, Desnoyers S, Zeng Z, Beidler DR, Poirier GG, Salvesen GS and Dixit VM: Yama/CPP32 beta, a mammalian homolog of CED-3, is a CrmA-inhibitable protease that cleaves the death substrate poly(ADP-ribose) polymerase. Cell 81: 801-809,1995.

18. Adams JM and Cory S: Life-or-death decisions by the Bcl-2 protein family. Trends Biochem Sci 26: 61-66, 2001.
19. Kim MS, Lee EJ, Kim HR and Moon A: p38 kinase is a key signaling molecule for H-Ras-induced cell motility and invasive phenotype in human breast epithelial cells. Cancer Res 63: 5454-5461, 2003.

20. Moon A, Kim MS, Kim TG, Kim SH, Kim HE, Chen YQ and Choi-Kim HR: H-ras, but not N-ras, induces an invasive phenotype in human breast epithelial cells: a role for MMP-2 in the H-rasinduced invasive phenotype. Int J Cancer 85: 176-181, 2000.

21. Shin I, Kim S, Song H, Kim HR and Moon A: H-Ras-specific activation of Rac-MKK3/6-p38 pathway: its critical role in invasion and migration of breast epithelial cells. J Biol Chem 280: 14675-14683, 2005.

22. Gebhardt C, Breitenbach U, Tuckermann JP, Dittrich BT, Richter KH and Angel P: Calgranulins S100A8 and S100A9 are negatively regulated by glucocorticoids in a c-Fos-dependent manner and overexpressed throughout skin carcinogenesis. Oncogene 21: 4266-4276, 2002.

23. Ott HW, Lindner H, Sarg B, Mueller-Holzner E, Abendstein B, Bergant A, Fessler S, Schwaerzler P, Zeimet A, Marth C and Illmensee K: Calgranulins in cystic fluid and serum from patients with ovarian carcinomas. Cancer Res 63: 7507-7514, 2003.

24. Arai K, Teratani T, Kuruto-Niwa R, Yamada T and Nozawa R: S100A9 expression in invasive ductal carcinoma of the breast: S100A9 expression in adenocarcinoma is closely associated with poor tumour differentiation. Eur J Cancer 40: 1179-1187, 2004.

25. Yong HY and Moon A: Roles of calcium-binding proteins, S100A8 and S100A9, in invasive phenotype of human gastric cancer cells. Arch Pharm Res 30: 75-81, 2007.

26. Reed JC: Bcl-2 family proteins. Oncogene 17: 3225-3236, 1998.

27. Mannello F, Tonti G and Papa S: Matrix metalloproteinase inhibitors as targets of anticancer therapeutics. Curr Cancer Drug Targets 5: 285-298, 2005.

28. Ray JM and Stetler-Stevenson WG: The role of matrix metalloproteases and their inhibitors in tumor invasion, metastasis and angiogenesis. Eur Respir J 7: 2062-2072, 1994.

29. Tsuchiya A, Kikuchi Y, Ando Y, Yoshida T and Abe R: Lymph node metastases in gastric cancer invading the submucosal layer. Eur J Surg Oncol 21: 248-250, 1995.

30. Yasuda K, Shiraishi N, Suematsu T, Yamaguchi K, Adachi Y and Kitano S: Rate of detection of lymph node metastasis is correlated with the depth of submucosal invasion in early stage gastric carcinoma. Cancer 85: 2119-2123, 1999.

31. Hermani A, Hess J, De servi B, Medunjanin S, Grobholz R, Trojan L, Angel P and Mayer D: Calcium-binding proteins S100A8 and S100A9 as novel diagnostic markers in human prostate cancer. Clin Can Res 11: 5146-5152, 2005.

32. Vogl T, Ludwing S, Geobeler M, Strey A, Thorey IS, Reichelt R, Foell D, Gerke V, Manitz MP, Nacken W, Werner S, Sorg C and Roth J: MRP8 and MRP14 control microtubule reorganization during transendothelial migration of phagocytes. Blood 104: 4260-4268, 2004.

33. Moon A, Yong HY, Song JI, Cukovic D, Salagrama S, Kaplan D, Putt D, Kim H, Dombkowski A and Kim HR: Global gene expression profiling unveils S100A8/A9 as candidate markers in H-ras-mediated human breast epithelial cell invasion. Mol Cancer Res 6: 1544-1553, 2008.

34. Yang J, Liu X, Bhalla K, Kim CN, Ibrado AM, Cai J, Peng TI, Jones DP and Wang X: Prevention of apoptosis by Bcl-2: release of cytochrome c from mitochondria blocked. Science 275: 1129-1132, 1997.

35. Jurgensmeier JM, Xie Z, Deveraux Q, Ellerby L, Bredesen D and Reed JC: Bax directly induces release of cytochrome $\mathrm{c}$ from isolated mitochondria. Proc Natl Acad Sci USA 95: 4997-5002, 1998.

36. Lin TH, Chang SJ, Chen CC, Wang JP and Tsao LT: Two phenanthraquinones from Dendrobium moniliforme. J Nat Prod 64: 1084-1086, 2001.

37. Lin SY, Chang YT, Liu JD, Yu CH, Ho YS, Lee YH and Le WS: Molecular mechanisms of apoptosis induced by magnolol in colon and liver cancer cells. Mol Carcinog 32: 73-83 2001.

38. Tominaga K, Higuchi K, Sasaki E, Suto R, Watanabe T, Fujiwara Y, Oshitani N, Matsumoto T, Kim S, Iwao H and Arakawa T: Correlation of MAP kinases with COX-2 induction differs between MKN45 and HT29 cells. Aliment Pharmacol Ther 20: 143-150, 2004.

39. Ji F, Chen YL, Jin EY, Wang WL, Yang ZL and Li YM: Relationship between matrix metalloproteinase-2 mRNA expression and clinicopathological and urokinase-type plasminogen activator system parameters and prognosis in human gastric cancer. World J Gastroenterol 11: 3222-3226, 2005. 